\title{
Entérovirus D68 : un virus à haut potentiel pathogène et épidémique
}

\author{
Audrey Mirand 1,2 \\ Jean-Luc Bailly ${ }^{1,2}$ \\ Hélène Peigue-Lafeuille ${ }^{1,2}$ \\ Cécile Henquell ${ }^{1,2}$ \\ ${ }^{1} \mathrm{CHU}$ Clermont-Ferrand, Laboratoire \\ de virologie, Centre national \\ de référence des entérovirus \\ et des parechovirus, \\ Laboratoire associé, \\ 63003 Clermont-Ferrand cedex, France \\ 2 Université Clermont-Auvergne, LMGE \\ UMR CNRS 6023, Équipe EPIE, \\ Épidémiologie et physiopathologie \\ des infections à entérovirus, \\ 63000 Clermont-Ferrand, France \\ <amirand@ chu-clermontferrand.fr>
}

\begin{abstract}
Résumé. L'entérovirus D68 (EV-D68), isolé pour la première fois en 1962, a longtemps été l'un des entérovirus les moins souvent détectés par les systèmes de surveillance à travers le monde. En 2014, des phénomènes épidémiques anormaux touchant des enfants aux États-Unis conduisent le Center for Disease Control and Prevention (CDC) à lancer deux alertes consécutives, suivies d'une description de nombreux cas dans d'autres pays. L'EV-D68 est responsable d'infections respiratoires sévères chez l'enfant et chez l'adulte, particulièrement en cas de maladie respiratoire sous-jacente. Des complications neurologiques peuvent survenir, proches de celles liées aux poliovirus ou à l'EV-A71. Les observations cliniques et radiologiques recueillies ont cependant permis d'identifier une entité neurologique spécifique associée à l'EV-D68, la myélite flasque aiguë, dont l'évolution est marquée de séquelles fréquentes. L'épidémiologie moléculaire des souches isolées dans différents pays du monde montre une évolution rapide du virus depuis les années 1990, témoignant d'une circulation importante dans la population. L'introduction de nouveaux clades pourrait expliquer la survenue d'épidémies dans des populations non immunisées. L'émergence récente de l'EV-D68 souligne encore une fois le pouvoir épidémique imprévisible des entérovirus et leur caractère neurotrope. Elle doit inciter à renforcer la surveillance de ces infections et à rechercher puis caractériser les entérovirus dans tous les tableaux infectieux sévères.
\end{abstract}

Mots clés : EV-D68, infection respiratoire, myélite flasque aiguë, émergence, réseaux de surveillance

\begin{abstract}
Since its discovery in 1962, enterovirus D68 (EV-D68) was one of the less frequently detected enteroviruses by the surveillance networks worldwide. In 2014, US pediatric hospitals reported increases in the number of children with severe respiratory illness. Following the alerts from the Center for Disease Control and Prevention, numerous cases of EV-D68 were reported in many countries. EVD68 is associated with severe respiratory infections in children and adults, mostly in patients with underlying respiratory diseases. Like with poliovirus and EVA71, rare but severe neurological complications may occur: acute flaccid myelitis is characterized by rapid onset of weakness and distinct abnormalities of the spinal cord gray matter on magnetic resonance imaging. Molecular epidemiology of strains isolated worldwide since the 90s shows a rapid evolution of the virus, reflecting its wide circulation in the general population. The recent emergence of EV-D68 underlines the unpredictable epidemic properties and the neurotropism of enteroviruses.
\end{abstract}

Key words: enterovirus D68, respiratory infection, acute flaccid myelitis, emergence, surveillance networks 


\section{Introduction}

Après sa découverte en 1962 en Californie, l'entérovirus D68 (EV-D68) a été responsable de foyers restreints d'infections respiratoires jusqu'en 2014, année au cours de laquelle plus de 2000 cas d'infections étaient rapportés [1]. L'histoire de la réémergence de ce virus commence en août 2014 quand deux hôpitaux américains des États du Missouri et d'Illinois rapportent une recrudescence d'hospitalisations pour infections respiratoires sévères associées à l'EV-D68 [2]. Le 26 septembre 2014, une deuxième alerte était lancée par le Center for Disease Control (CDC) après la notification le 12/09/2014 par un hôpital du Colorado d'un groupe de neuf enfants présentant une atteinte neurologique sévère caractérisée par une faiblesse des membres et des atteintes des paires crâniennes [3]. Cette alerte faisait écho à l'annonce faite en février 2014 de la survenue de plusieurs cas de maladie « polio-like » entre 2012 et 2014 en Californie, avec un EV-D68 détecté dans les sécrétions respiratoires chez $48 \%$ des patients [4]. Fin septembre 2014, le CDC demandait la notification rétrospective (au $1^{\text {er }}$ août 2014) et prospective des cas d'atteintes neurologiques définies par «la survenue brutale d'une faiblesse des membres avec des lésions restreintes de la substance grise de la moelle épinière en imagerie de résonance magnétique (IRM) chez les patients de moins de 21 ans » [3]. Sur la base des données cliniques et radiologiques, cette entité sera désignée plus tard sous le terme de «myélite flasque aiguë » (MFA) ou Acute Flaccid Myelitis [5] pour la distinguer des autres formes de paralysies flasques aiguës [6]. Suite à ces deux alertes, de nombreux cas d'infections respiratoires, dont certaines sévères, étaient rapportées dans plusieurs pays européens [7] dont la France où une surveillance spécifique a été mise en place début octobre 2014 [8]. Le premier cas européen de MFA associé à une infection à EV-D68 était rapporté en France chez un enfant hospitalisé à Clermont-Ferrand fin septembre 2014 [9].

Cette épidémie d'infections à EV-D68 avec atteintes respiratoires sévères et complications neurologiques, jamais décrites jusque-là pour ce virus, n'est pas sans rappeler celles associées à l'EV-A71 (épidémies de maladie pieds-mains-bouche associées à des rhombencéphalites) en Asie depuis la fin des années 90 [10] et place l'EVD68 sur la liste noire des EV à surveiller. La surveillance mise en place aux États-Unis et dans plusieurs pays européens a d'ailleurs montré une circulation continue de l'EV-D68 depuis 2014 et une recrudescence des infections respiratoires associées à des paralysies en 2016 [11-16].

\section{Un entérovirus pas comme les autres?}

L'EV-D68 a été isolé pour la première fois à partir d'échantillons respiratoires de quatre enfants présentant une pneumonie (USA, Californie, 1962) [17]. Ce picornavirus appartient à l'espèce taxonomique $\mathrm{D}$ du genre Enterovirus (EV) avec quatre autres types (EV-D70, EV-D94, EV-D111 et EV-D120). Sur la base du degré de parenté génétique entre les séquences codant les protéines de capside et en particulier celle codant la protéine majeure de capside VP1, le genre Enterovirus comprend également trois autres espèces d'EV ( $\mathrm{A}$ à $\mathrm{C}$ ), trois espèces de rhinovirus (RV) (A à $\mathrm{C}$ ) et cinq espèces d'EV animaux (E à J, http://www.picornaviridae.com, au 01/12/2017). Comme les autres EV, l'EV-D68 est un petit virus nu dont la capside icosaédrique comprend quatre protéines structurales VP1 à VP4 (figure 1A). Le génome est un ARN simple brin d'environ 7300 nucléotides et se compose de trois régions fonctionnelles : la région $5^{\prime}$ non codante, un cadre de lecture ouvert codant une polyprotéine et une région $3^{\prime}$ non codante (figure $1 B$ ). La polyprotéine est clivée par des protéases virales $\left(2 \mathrm{~A}^{\text {pro }}\right.$ et $\left.3 \mathrm{C}^{\text {pro }}\right)$ en trois précurseurs protéiques (P1-P3) à partir desquels sont formées les quatre protéines structurales (segment P1) et les sept protéines non structurales (segments P2 et P3) [18].

L'EV-D68 présente des caractéristiques physico-chimiques et structurales communes avec les rhinovirus : sa température optimale de réplication est de $33^{\circ} \mathrm{C}$ (versus $37^{\circ} \mathrm{C}$ pour les autres EV) et il est sensible à la chaleur et à un $\mathrm{pH}$ acide [19]. La face externe de la capside des EV présente une profonde dépression (appelée canyon) qui entoure le sommet icosaédrique pentagonal et constitue un site de liaison aux récepteurs cellulaires. Chez l'EV-D68 et les rhinovirus, le canyon est moins profond et plus étroit [20]. Des analyses structurales et fonctionnelles ont montré que les acides sialiques étaient des récepteurs fonctionnels pour l'EV-D68 $[21,22]$ car la liaison à ces récepteurs modifie la conformation de la capside nécessaire au processus de décapsidation [22]. La présence des acides sialiques à la surface des cellules épithéliales respiratoires peut expliquer le tropisme respiratoire de l'EV-D68. Imamura et coll [pour revue, 23] ont montré que ce virus avait une plus forte affinité pour les acides sialiques liés en $\alpha$ 2,6 exprimés notamment dans la partie supérieure du tractus respiratoire et qui sont également les récepteurs des virus grippaux humains. L'EV-D68 peut se lier à des récepteurs sialylés sans spécificité vis-à-vis d'une protéine particulière et certaines souches circulantes sont capables d'infecter des cellules indépendamment de la présence d'acides sialiques. De plus, le «Decay Accelerating Factor » ou DAF, également utilisé par l'EV-D70, certains ECHOvirus et largement distribué 


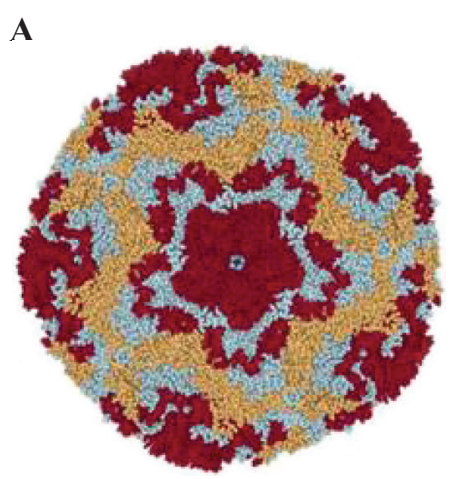

B

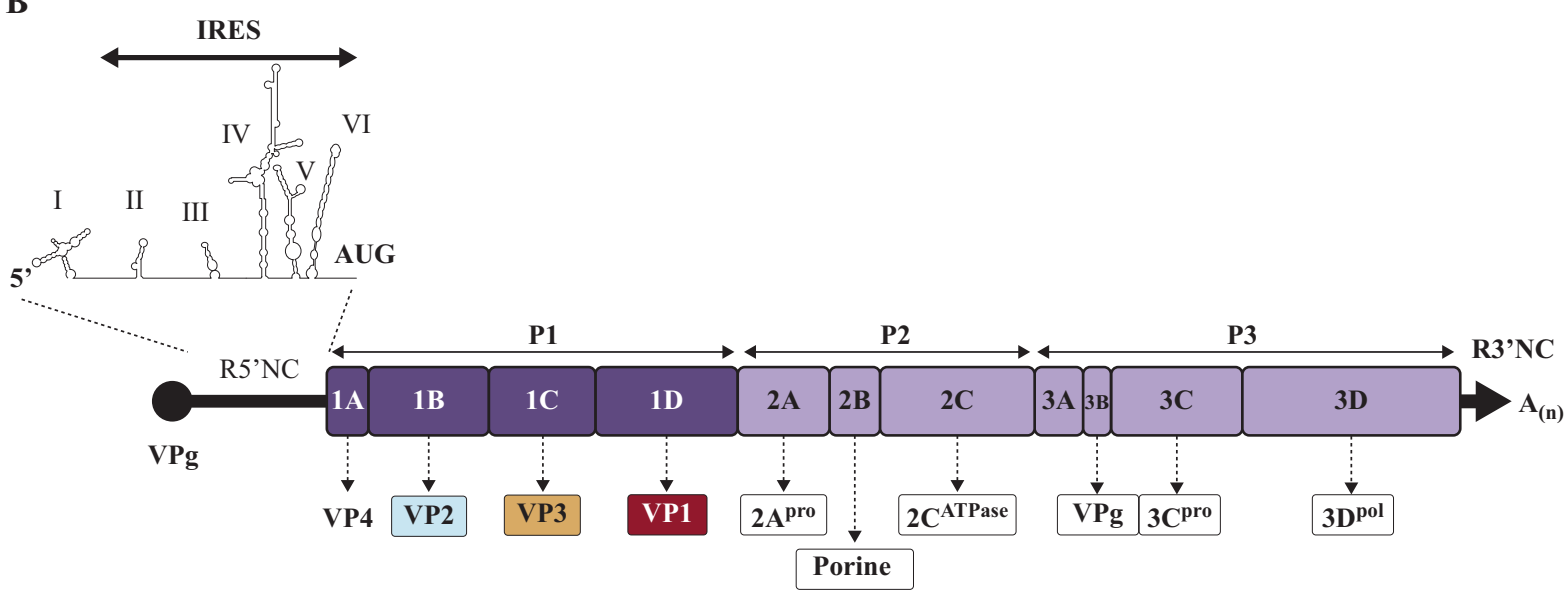

Vivirologi̊日e

Figure 1. Structure tridimensionnelle de la capside de l’EV-D68 (A) et organisation fonctionnelle du génome des entérovirus (B). (A). La capside est constituée des protéines VP1 (rouge foncé), VP2 (orange), VP3 (bleu ciel) exposées à la surface et de la protéine VP4 sur la face interne. La structure tridimensionnelle de la capside de l'EV-D68 a été représentée à partir des données enregistrées dans la banque de séquences protéiques RCSB (https://www.rcsb.org ; Numéro d'identification 3WM8) d'après les travaux de Liu et al. [20]. (B). Le génome se compose d'un long cadre de lecture ouvert encadré par deux régions non codantes à chaque extrémité $5^{\prime}$ et $3^{\prime}$. La région $5^{\prime}$ non codante est liée à une petite protéine (VPg) qui est, après pénétration du virus dans la cellule, libérée par clivage pour donner un ARNm fonctionnel. Cette région est également caractérisée par de nombreuses structures secondaires constituant le site interne d'initiation de la traduction ou IRES et intervenant dans la traduction et la réplication virale. Après traduction, la maturation de la polyprotéine est assurée par les protéases virales $2 A^{\text {pro }}$ et $3 C^{\text {pro }}$ pour générer l'ensemble des protéines fonctionnelles.

Abréviations : R5' NC, région 5' non codante ; R3'NC, région 3' non codante ; IRES, Internal Ribosome Entry Site.

au niveau des cellules épithéliales du tractus respiratoire inférieur serait également un récepteur pour l'EV-D68 [24]. Ces données suggèrent la possibilité pour ce virus d'utiliser des récepteurs multiples [21] qui restent encore à découvrir.

La survenue de complications neurologiques à type de myélites flasques aiguës dans un contexte d'épidémie d'infections respiratoires associées à l'EV-D68 pose la question d'un éventuel lien étiologique. D'abord controversés, le neurotropisme et la neurovirulence de cet $\mathrm{EV}$ ont été démontrés de façon expérimentale. Un modèle murin a permis de montrer que les souches circulantes, contrairement à la souche prototype Fermon, étaient capables d'induire une paralysie, caractérisée par une destruction des neurones moteurs de la moelle épinière, de façon similaire au cas de MFA [25]. Trois observations réalisées chez la souris ont confirmé le rôle direct du virus dans la paralysie : (1) la détection de l'EV-D68 dans les neurones moteurs, (2) l'induction d'une paralysie chez des souris naïves après inoculation du virus issu de la moelle épinière de souris 
Tableau 1 Principales données cliniques et diagnostiques des infections à EV-D68.

\begin{tabular}{|c|c|c|c|}
\hline & Symptômes cliniques & Diagnostic & Traitement \\
\hline Atteintes respiratoires & $\begin{array}{l}\text { Rhinite simple à des formes } \\
\text { sévères (pneumonies, détresse } \\
\text { respiratoire aiguë) } \\
\text { Infection sévère plus fréquente } \\
\text { chez les patients ayant des } \\
\text { antécédents d'asthme ou une } \\
\text { pathologie pulmonaire chronique }\end{array}$ & $\begin{array}{l}\text { Aspiration ou écouvillonnages } \\
\text { naso-pharyngés ou lavage } \\
\text { broncho-alvéolaire } \\
\text { Détection génomique } \\
\text { des entérovirus } \\
\text { et/ou des rhinovirus }\end{array}$ & \multirow{2}{*}{ Symptomatique } \\
\hline $\begin{array}{l}\text { Atteinte neurologique } \\
=\text { myélite flasque aiguë }\end{array}$ & $\begin{array}{l}\text { Enfants } \\
\text { Prodromes : fièvre, syndrome } \\
\text { pseudo-grippal, signes digestifs, } \\
\text { myalgies } \\
\text { Survenue brutale d'une faiblesse } \\
\text { des membres associée à des } \\
\text { lésions restreintes à la substance } \\
\text { grise sur un ou plusieurs } \\
\text { segments à l'IRM médullaire } \\
\text { Signes spécifiques associés à } \\
\text { une atteinte des paires } \\
\text { crâniennes } \\
\text { Séquelles motrices fréquentes }\end{array}$ & $\begin{array}{l}\text { Liquide céphalo-rachidien : } \\
\text { pléiocytose (> cinq leucocytes } / \mathrm{mm}^{3} \text { ) } \\
\text { fréquente mais modérée, } \\
\text { protéinorrachie normale ou } \\
\text { légèrement augmentée } \\
\text { Détection génomique des } \\
\text { entérovirus et/ou des rhinovirus } \\
\text { - Aspiration ou écouvillonnages } \\
\text { naso-pharyngés } \\
\text { Détection du génome des entérovirus } \\
\text { - LCR : détection rare du génome } \\
\text { - Ecouvillonnage de gorge } \\
\text { - Selles : détection rare du génome } \\
\text { - Sang : chez les jeunes enfants }\end{array}$ & \\
\hline
\end{tabular}

infectées et (3) la protection contre l'infection conférée par un sérum anti EV-D68. Chez ces souris, l'injection intramusculaire ou intranasale induit une paralysie des membres antérieurs et au niveau du site d'injection pour la voie intramusculaire, suggérant une infection par transport axonal [25]. Cependant, une virémie a été démontrée chez des enfants présentant une pneumonie [23] et l'EV-D68 est capable d'infecter les leucocytes et les cellules endothéliales in vitro [26]. La dissémination intra individuelle de l'EV-D68, comme celle des poliovirus et de l'EV-A71, utiliserait donc à la fois la voie sanguine et la voie axonale, mais les mécanismes qui facilitent l'une ou l'autre voie sont à élucider. La protéine ICAM-5, un facteur d'adhésion intercellulaire spécifique des neurones également appelé télencephaline, pourrait constituer le récepteur de l'EVD68 dans le système nerveux [27].

Aucun facteur de virulence n'a pour l'instant été retrouvé parmi les souches d'EV-D68 associées à des formes paralytiques. Une même souche peut être isolée chez un patient atteint d'infection respiratoire simple ou un patient atteint de paralysie. De même, si les patients souffrant de pathologies respiratoires chroniques sont plus à risque de développer une infection respiratoire sévère, les complications neurologiques n'y sont pas associées. D'autres facteurs liés à l'hôte pourraient cependant être impliqués dans la neuropathogénèse de l'infection. De nombreuses études sont encore à réaliser pour déterminer s'il existe des facteurs viraux ou liés à l'hôte et quelle est leur part relative dans la survenue d'une MFA.

\section{Principales manifestations cliniques}

Les données cliniques collectées au cours des épidémies d'infections à EV-D68 en 2014 ont permis de préciser le spectre des manifestations cliniques associées à ce virus (tableau 1).

\section{Infections respiratoires}

L'EV-D68 est responsable d'un large éventail de pathologies respiratoires depuis la simple rhinite aux formes plus sévères telles que pneumonie ou détresse respiratoire aiguë $[1,8,23,28]$. Aux États-Unis, en 2014, les infections respiratoires ont affecté essentiellement les enfants âgés de un à 11 ans (trois jours-92 ans ; âge médian = cinq ans). Les principaux symptômes cliniques observés étaient une dyspnée $(84 \%)$, la toux $(81 \%)$ et une sibilance $(70 \%)$. La fièvre était présente chez $48 \%$ des patients à l'admission. Plus de la moitié des enfants avaient des antécédents d'asthme ou d'hyperréactivité bronchique mais des râles sibilants ont aussi été observés chez des enfants sans antécédents [28]. En France, le diagnostic final retenu était l'asthme ou l'exacerbation d'asthme dans $48 \%$ des cas ou la bronchiolite dans $21 \%$ [8]. L'épidémie américaine en 2014 a été marquée par un taux important d'infections sévères avec près des deux-tiers des patients admis en soins intensifs et $28 \%$ ayant dû bénéficier d'une assistance ventilatoire [28]. Ces chiffres sont cependant à nuancer car il est probable que la collection des échantillons et la recherche de 
l'EV-D68 aient été plus fréquemment réalisées chez les enfants présentant une infection sévère. Dans d'autres études, la même année, le taux d'admission en soins intensifs était en effet compris entre 6,8 et $27 \%[8,29]$. Les patients asthmatiques ou présentant une hyperréactivité bronchique ont davantage de risques de développer une infection sévère $[28,30]$. Par rapport aux infections respiratoires dues aux autres EV-Rhinovirus, l'infection à EV-D68 semble plus fréquemment associée à une détresse respiratoire [29].

L'impact clinique des infections à EV-D68 chez les adultes est sûrement sous-estimé, la recherche des infections à Rhinovirus-EV étant plus fréquemment réalisée en pratique courante chez les enfants. Dans une étude néerlandaise d'échantillons respiratoires collectés dans le cadre de la surveillance de la grippe en 2010, les infections à EVD68 étaient plus fréquentes chez les patients de 50 à 59 ans que chez les enfants de moins de dix ans [31]. Comme chez les enfants, les adultes ayant une pathologie respiratoire sous-jacente ou les patients immunodéprimés ont un risque plus élevé d'infection sévère [8].

\section{Complications neurologiques: la myélite flasque aiguë}

D'après les connaissances actuelles, les complications neurologiques associées aux infections à EV-D68 se présentent surtout sous la forme d'une myélite flasque aiguë (MFA) et ont été presque exclusivement rapportées au cours des épidémies d'infections respiratoires à EV-D68. Avant 2014, deux cas d'atteintes neurologiques sévères associées à l'EV-D68 (avec détection du génome dans le liquide céphalo-rachidien) avaient été décrits en 2005 [32] et en 2008 [33]. Entre août et décembre 2014, 120 cas ont été recensés suite à la demande de notification par le CDC aux États-Unis qui ciblait les patients de moins de 21 ans. L'âge médian des patients était de sept ans (trois mois-21 ans). La même année en Europe, quatre cas étaient recensés dont deux en France [9, 34] et deux en Norvège [35], tous étaient des enfants de moins de six ans. En 2016, la recrudescence des infections à EV-D68 dans de nombreux pays [11-16] s'est aussi accompagnée d'une augmentation des cas de MFA avec 144 cas aux États-Unis (https://www.cdc.gov/acute-flaccidmyelitis/afm-surveillance.html, consulté le 01/12/2017) et neuf en France [11]. Depuis le $1^{\text {er }}$ août 2015, une définition a été établie par les autorités américaines afin de faciliter les déclarations de cas de MFA : un cas confirmé est défini par la survenue brutale d'une faiblesse des membres associée à des images d'IRM médullaire montrant des lésions restreintes à la substance grise sur un à plusieurs segments. En l'absence d'imagerie, le cas est considéré comme probable si une pléiocytose du liquide céphalo-rachidien (LCR, > cinq leucocytes $/ \mathrm{mm}^{3}$ ajusté sur la présence d'hématies en soustrayant un leucocyte pour 500 hématies) est associée à la faiblesse des membres [5, https://www.cdc.gov/acute-flaccidmyelitis/hcp/case-definition.html, mise à jour 02/03/2017, consultée le 01/12/2017].

La MFA est caractérisée par la survenue brutale d'une paralysie flasque et asymétrique des membres associée à une perte des réflexes et une absence de déficit sensoriel. La paralysie survient sept jours (1-16 jours) après un épisode infectieux caractérisé par de la fièvre, des symptômes respiratoires et/ou digestifs et des myalgies [9, 36, 37]. La notion de contage familial est fréquente. Une évolution en deux phases est très souvent rapportée avec une amélioration du syndrome infectieux avant le retour de la fièvre, puis un syndrome méningé suivi des déficits neurologiques progressant en quelques heures. Les muscles proximaux des membres supérieurs sont les plus souvent atteints bien qu'une tétraparésie soit fréquemment observée [9, 36, 37]. Une atteinte des paires crâniennes est observée chez 25 à $50 \%$ des patients et se traduit par une diplopie, une dysphagie ou une paralysie faciale [36]. Les données d'imagerie et d'électromyographie montrent l'atteinte des neurones moteurs et sont prises en compte dans la définition des cas de MFA [38]. Si ces données d'imagerie sont parfois très similaires à celles associées aux paralysies flasques aiguës dues aux poliovirus, à l'EV-A71 [39] ou à l'infection par le virus West Nile, certaines caractéristiques des atteintes neurologiques associées à l'EV-D68 les en différencient : 1) l'atteinte fréquente des membres supérieurs ou des nerfs crâniens (rare dans la poliomyélite), 2) l'absence de complications cardiopulmonaires similaires à celles décrites avec l'EV-A71 [10], 3) l'absence de lésions sus-tentorielles et d'encéphalites (virus West Nile et EV-A71) [10]. Il est intéressant de noter ici, qu'au cours de certaines atteintes neurologiques associées à l'EV-D68, des signes d'atteinte cardiaque (myocardite, péricardite, insuffisance cardiaque aiguë) ont été observés $[9,11]$, rappelant le cardiotropisme de certains EV.

Comme pour l'EV-A71, les séquelles neurologiques, en particulier motrices, sont très fréquentes. Un suivi réalisé sur un an de huit des 12 enfants de la première cohorte décrite aux États-Unis [37] a montré que 6/8 (75\%) présentaient des déficits moteurs prédominant au niveau des muscles proximaux des membres supérieurs associés à une atrophie [40]. Une résolution complète des déficits a été observée pour deux enfants après six et neuf mois d'évolution. Lorsque les membres inférieurs sont atteints initialement, une faiblesse persiste à un an malgré une amélioration progressive des fonctions motrices et des symptômes associés aux atteintes des paires crâniennes [40]. 


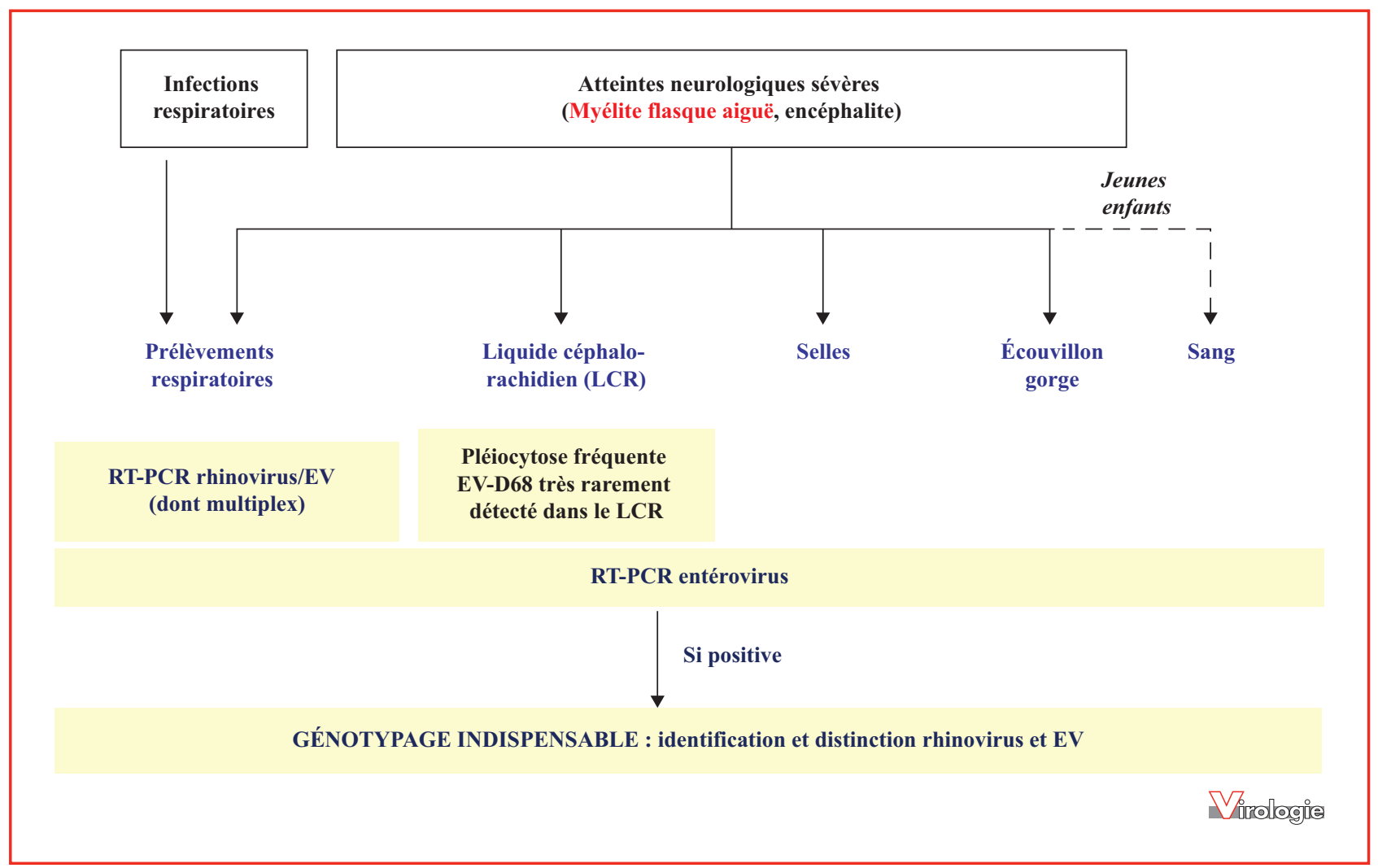

Figure 2. Diagnostic virologique des infections à EV-D68.

Le génotypage est recommandé pour toute atteinte sévère (respiratoire ou neurologique).

\section{Diagnostic virologique}

La recherche des EV doit être réalisée devant toute infection respiratoire sévère, en particulier chez les patients admis en soins intensifs. Les infections respiratoires à EV-D68 ne se distinguent pas des autres causes d'infections respiratoires [29]. De plus, l'absence fréquente de fièvre à l'admission rend difficile leur distinction d'une exacerbation d'asthme associée à une cause non infectieuse. En règle générale, toute atteinte neurologique sévère doit inciter à rechercher les EV en parallèle des autres agents étiologiques possibles.

Le diagnostic repose sur la détection du génome des EV dans les échantillons cliniques. En cas d'atteinte respiratoire isolée, un prélèvement respiratoire (écouvillon nasopharyngé avec milieu de transport, aspiration nasopharyngée, lavage broncho-alvéolaire) réalisé le plus précocement possible après le début des signes cliniques est suffisant. En cas d'atteintes neurologiques, il est nécessaire de multiplier les prélèvements pour augmenter les chances de détecter les EV ou un autre agent (figure 2). Toute manifestation neurologique dans un contexte infectieux doit conduire à la réalisation d'une ponction lombaire. $\mathrm{Si}$ le LCR constitue le prélèvement de choix pour le diag- nostic de certitude d'une méningite à EV [41], le génome des EV est cependant très rarement détecté dans les atteintes neurologiques incriminant l'EV-A71 ou l'EV-D68 [36], malgré une pléiocytose fréquente ( $>$ cinq leucocytes $/ \mathrm{mm}^{3}$ ) $[9,37]$. Dès l'admission du patient, il faut donc associer au LCR : un échantillon respiratoire (même en l'absence de prodromes respiratoires), un échantillon de selles dans lesquelles les EV sont excrétés pendant plusieurs semaines et un écouvillonnage de gorge. Le sang peut également être prélevé, en particulier chez les jeunes enfants [23]. Lors de l'investigation des MFA rapportées aux États-Unis en 2014, le taux de détection de l'EV-D68 dans les échantillons respiratoires était plus élevé que dans les selles ou le LCR mais diminuait en fonction du délai entre le début des signes cliniques et la réalisation du prélèvement : il était de $47 \%$ lorsque le prélèvement était réalisé tôt (dans les sept jours) contre $20 \%$ tout délai confondu [36].

La détection génomique est réalisée à l'aide de trousses ciblant spécifiquement la région 5' non codante des EV. Du fait de la parenté génétique élevée entre les EV et les rhinovirus (ils appartiennent au même genre Enterovirus), ils sont souvent détectés de façon indifférenciée par les tests du marché. La détection génomique peut être réalisée à l'aide de 
trousses spécifiques et génériques pour les entérovirus, les trousses commercialisées et utilisées en France permettant tout à fait la détection des EV-D68. Pour les prélèvements respiratoires, les trousses multiplex détectant un panel de virus respiratoires ou les trousses ciblant les rhinovirus sont elles aussi capables de détecter ce virus, leur sensibilité visà-vis de cet EV devant cependant être évaluée. Quelles que soient les trousses utilisées, elles ne permettent qu'un diagnostic de genre ; ainsi, dans les prélèvements respiratoires, le résultat est souvent rendu positif pour «Rhinovirus-EV ». Il est alors indispensable, en particulier dans les infections sévères, d'aller plus loin en génotypant le virus détecté pour identifier son type et faire la distinction entre rhinovirus et $\mathrm{EV}$ pour les prélèvements respiratoires. Ce génotypage cible le plus souvent les séquences codant les protéines de capside VP4 et VP2 et peut être réalisé sur place ou transmis à l'un des deux laboratoires du Centre National de Référence des Enterovirus et Parechovirus à Lyon ou Clermont-Ferrand qui le réalise à titre gracieux [18]. La recherche spécifique de l'EV-D68 pourra aussi être réalisée à l'aide d'une RT-PCR en temps réel (test 'maison', non commercialisé) [7], à titre diagnostique ou épidémiologique en cas de recrudescence d'atteintes neurologiques ou respiratoires sévères.

\section{Épidémiologie et émergence des infections à EV-D68}

Du fait de l'intérêt récent pour ce virus et du nombre restreint d'infections identifiées à EV-D68 avant 2014 à travers le monde, les données disponibles comprennent de nombreux biais (études rétrospectives, exploration uniquement des cas sévères), et notre connaissance de l'épidémiologie des infections à EV-D68 est encore parcellaire.

L'homme est le seul réservoir de ce virus. Le tropisme respiratoire de l'EV-D68 favorise une transmission par aérosol mais une transmission fécale-orale est possible car le virus peut être détecté dans les selles [9, 42]. Les infections à EV-D68 surviennent sur un mode épidémique, le plus souvent à la fin de l'été et au début de l'automne [8, 23, 28] avant la période habituelle des épidémies d'infections respiratoires à virus respiratoire syncitial ou aux virus grippaux. Comme pour les infections à EV en général, des cas sont possibles en hiver. En France, en 2014, si deux pics de détection de l'EV-D68 ont été observés en octobre (semaine 43) et en novembre (semaine 48), ce virus a été détecté sporadiquement jusqu'à la fin de l'année [8].

L'EV-D68 n'a réellement suscité l'intérêt qu'en 2014, suite aux alertes américaines, bien qu'il soit considéré comme un pathogène émergent depuis 2008 [23]. Cette émergence pourrait être en partie liée à l' amélioration du diagnostic des infections respiratoires avec la commercialisation récente de nombreuses trousses permettant la détection génomique des rhinovirus dans les prélèvements respiratoires.

En l'absence de surveillance des infections respiratoires spécifiquement associées à l'EV-D68 qui nécessiterait un génotypage exhaustif des échantillons positifs à Rhinovirus-EV, il est difficile de déterminer s'il s'agit d'une réémergence réelle. Toutefois, des études rétrospectives réalisées au Japon et aux Pays-Bas ont mis en évidence une circulation continue mais sporadique de l'EV-D68 jusqu'en 2010, année pendant laquelle des épidémies ont été rapportées [31, 43]. Une étude chinoise de séroprévalence a montré une augmentation de la circulation du virus entre 2004 et 2011 à travers l'augmentation continue du titre en anticorps spécifiques [44].

Trois périodes peuvent être distinguées dans l'épidémiologie des infections à EV-D68. Entre 1962 et 2008, quelques infections à EV-D68 ont été rapportées aux États-Unis [32], aux Pays-Bas [31] et au Japon [43]. La deuxième période (2008-début 2014) marque le début de l'émergence de l'EV-D68. De petits foyers d'infections sont alors rapportés aux États-Unis, Asie et en Europe (figure 3) avec une recrudescence des infections observées en 2010 et la détection de l'EV-D68 dans 15 pays sur quatre continents entre 2010 et 2014 [pour revue, 1, 23]. Enfin, l'épidémie de 2014 représente la plus grande épidémie d'infections à EV-D68 avec près de 2287 cas confirmés dans le monde (dont 1153 aux États-Unis). Suite à l'alerte lancée par les CDC, de nombreuses infections ont été rapportées au Canada, en Europe (figure 3), en Asie (Taïwan, Thaïlande) et en Amérique du Sud (Brésil, Chili) [pour revue, 1]. La mise en place d'une surveillance renforcée et spécifique dans de nombreux pays depuis 2014 a permis de mettre en évidence la circulation continue de l'EV-D68 et de détecter des épidémies d'infections en 2016 aux États-Unis, en France, Espagne, Pays Bas, Danemark et Suède (figure 3) [11-16]. En France, à travers le réseau de surveillance des infections à EV (RSE), 205 cas ont été rapportés en 2014, seulement trois en 2015, puis 202 en 2016 (données du CNR).

\section{Un virus en évolution}

L'évolution des EV est régulièrement marquée par l'émergence de variants génétiques par une accumulation régulière de mutations ponctuelles et l'intervention de la recombinaison homologue entre souches de la même espèce. L'analyse des séquences géniques $1 \mathrm{D}$, codant la protéine de capside VP1, couplée à celle de régions génomiques distantes (codant pour des protéines non structurales, en 3' du génome viral) permet d'étudier l'évolution 


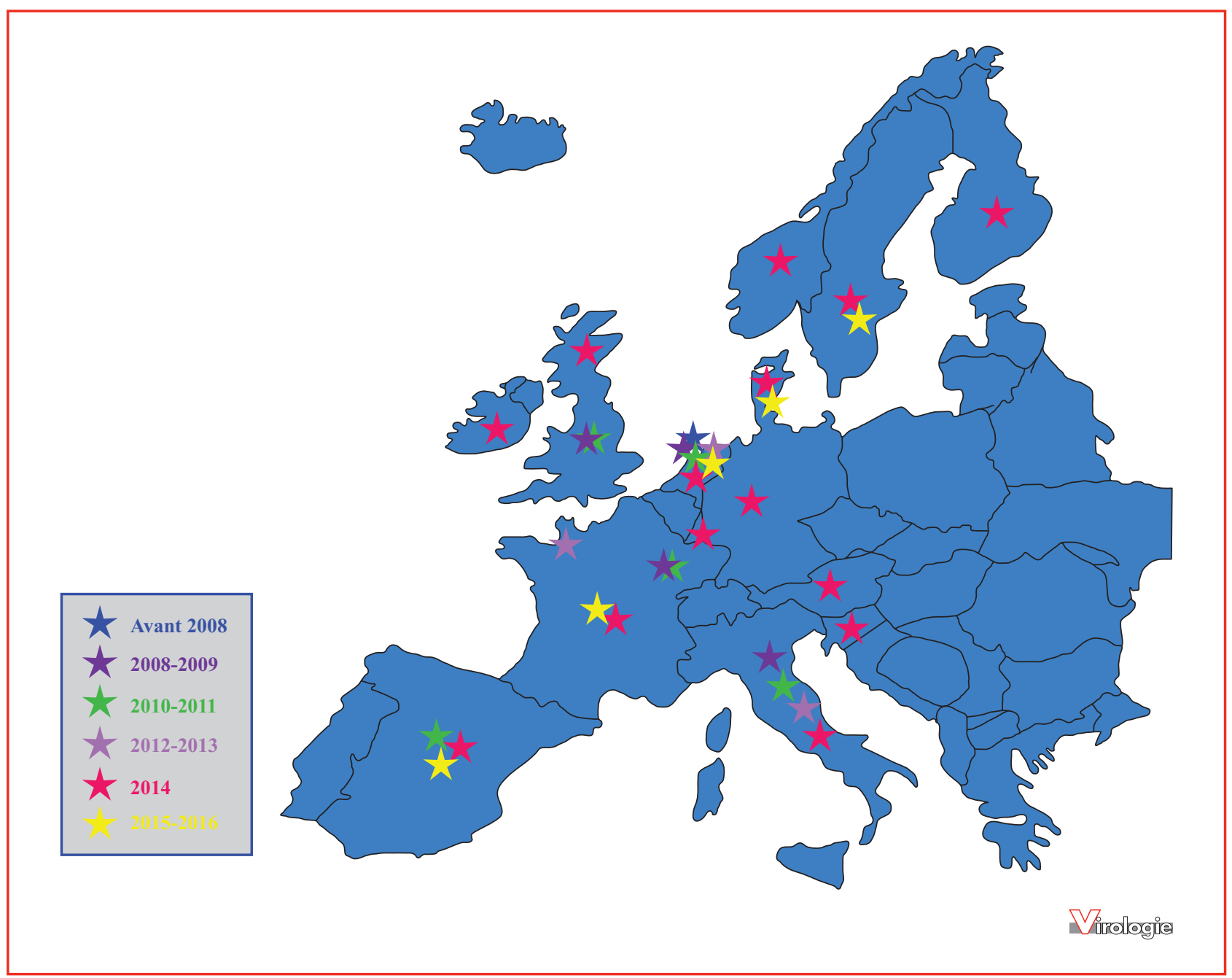

Figure 3. Infections à EV-D68 rapportées en Europe entre 1994 et 2016.

D'après références $[1,7,8,11-15,31]$.

des souches au cours du temps. L'analyse phylogénétique des séquences 1D permet notamment de distinguer des génogroupes ou sous-génogroupes bien que les seuils de différences nucléotidiques ne soient pas clairement établis pour définir ces classes intra-typiques. Pour l'EV-D68, un seul génogroupe, apparu autour de 1960, est reconnu sur la base des séquences 1D [45]. L'évolution rapide de cet EV, depuis le début des années 1990 [31], présente plusieurs caractéristiques. Tout d'abord, la phylogénie de l'EV-D68 montre l'existence de quatre clades ou sousgénogroupes $(\mathrm{A}-\mathrm{D})$. Au sein du clade $\mathrm{B}$ se différencient trois lignées B1 à B3 (figure 4) [45, 46]. De plus, chaque clade et lignée se distingue par des mutations spécifiques dans différentes régions génomiques et des indels (un ou deux codons) dans le gène $1 \mathrm{D}$, ce qui est rare entre des séquences d'un même type d'EV. Des substitutions d'acides aminés exposés à la surface du virus [20] ont notamment été observées dans la protéine de capside VP1, en particulier dans des boucles reliant les feuillets $\beta B$ et $\beta C$ et $\beta D$ et $\beta E$ (figure 5) [46]. Ces variations constituent des signatures phylogénétiques et affectent potentiellement des sites antigéniques [23].

L'analyse de génomes complets, représentant les clades $\mathrm{A}, \mathrm{C}$ et $\mathrm{D}$, a mis en évidence des différences à la fin de la région $5^{\prime}$ non codante [45], dans des sites de clivage des protéases $2 \mathrm{~A}$ et $3 \mathrm{C}$, et dans la région $3^{\prime}$ non codante. Ces variations pourraient avoir un impact sur la réplication virale et la transmission du virus [47]. Plus récemment, des évènements de recombinaison intra- (B1/B2) et inter-clades (A/D) ont également été identifiés dans les protéines de capside de deux souches d'EV-D68 [48]. Les conséquences fonctionnelles des mutations et des indels sur la réplication 


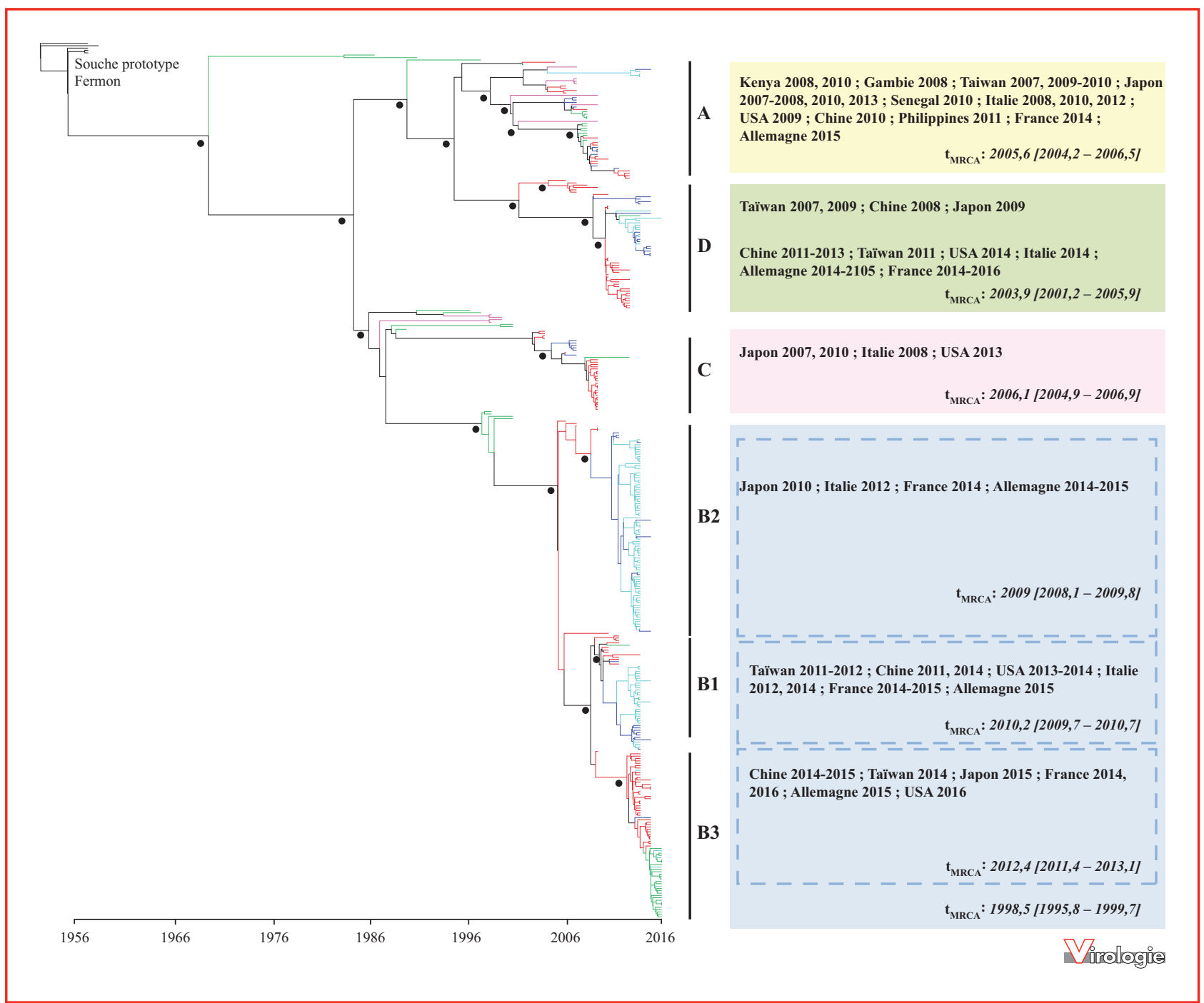

Figure 4. Arbre phylogénétique des séquences 1D complètes des souches d'EV-D68 ayant circulé entre 1962 et 2016.

Les relations de parenté génétique ont été analysées par méthode bayésienne à partir d'un échantillon de 376 séquences $1 \mathrm{D}$ non redondantes disponibles dans les banques de données au 25/07/2017. Chaque continent est représenté par une couleur : Afrique, rose ; Amérique du Nord, vert ; Europe, bleu foncé ; Asie, rouge. Les séquences des souches isolées en France sont indiquées en bleu clair. Les embranchements ayant une probabilité postérieure $>0,9$ sont indiqués par des ronds. Les dates estimées pour les ancêtres communs le plus récents $\left(t_{M R C A}\right)$ sont indiquées pour chaque clade.

virale doivent être confirmées par des études spécifiques. Comme dans le cas de l'EV-A71, le pouvoir pathogène de l'EV-D68 et la survenue d'une MFA ne semblent pas corrélés aux séquences génomiques virales [25].

Ces nombreuses variations génomiques témoignent surtout d'une transmission intense du virus. Elles peuvent avoir favorisé l'émergence de l'EV-D68 à une échelle globale au cours de ces 20 dernières années. L'absence d'immunité croisée entre les clades A, B et C [23] pourrait expliquer la survenue des infections à EV-D68 dans une population non encore immunisée vis-à-vis d'un clade en particulier. Cette hypothèse explique parfaitement la succession d'épidémies causées par des souches de clades différents. Dans ce cadre, l'émergence du clade B1 aux États-Unis serait ainsi liée à la faible circulation du clade $\mathrm{B}$ auparavant et à une absence d'immunité de la population générale. Au sein d'un même clade, bien que des mutations signatures aient été identifiées parmi les lignées B1 à B3, les conséquences sur les caractéristiques antigéniques des souches n'ont pas encore été étudiées. En 2016, le remplacement des lignées B1 et B2 aux États-Unis et en Europe par la lignée B3 reste inexpliqué. 


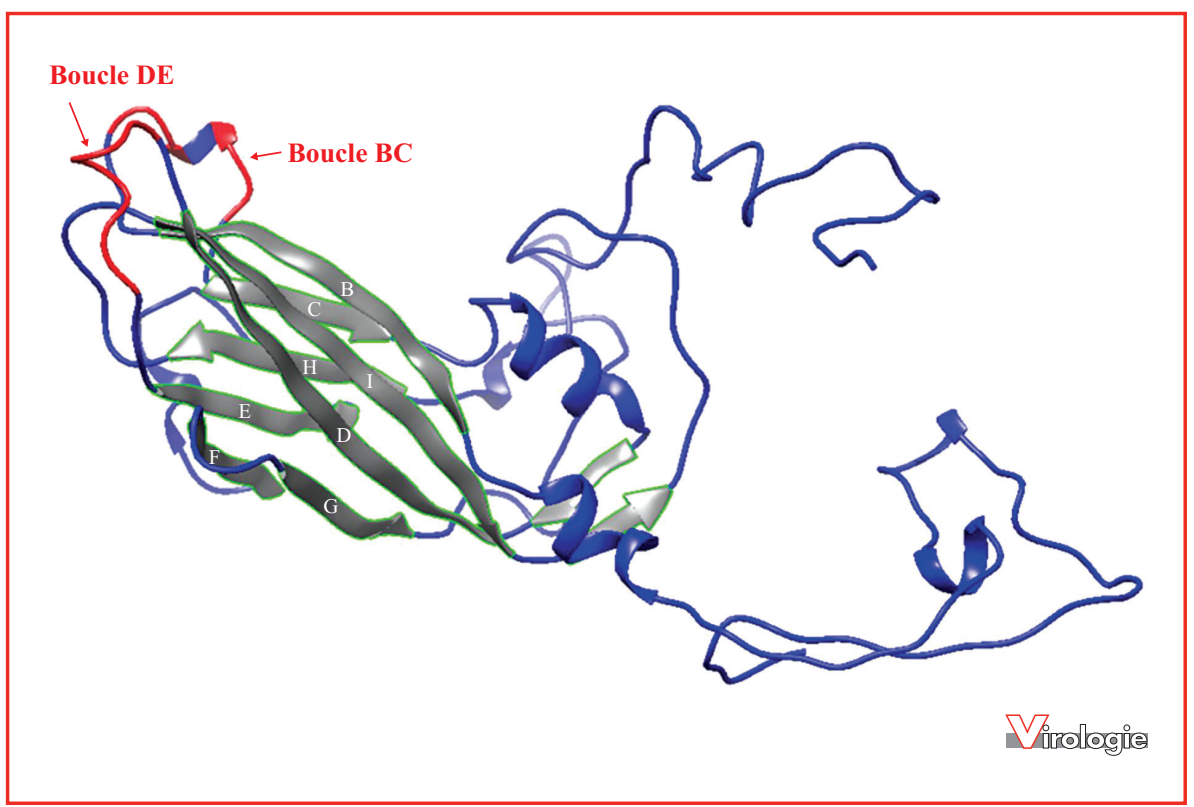

Figure 5. Représentation schématique en ruban de la protéine de capside VP1.

La structure en ruban de la protéine VP1 a été reconstruite à partir d'une séquence 1D d'une souche clinique isolée au CHU de ClermontFerrand en 2014 (numéro d'accession LN681336) à l'aide du logiciel Chimera (www.cgl.ucsf.edu/chimera). Les feuillets $\beta$ sont représentés en gris. Des boucles d'acides aminés connectent les différents feuillets et certaines comme les boucles BC et DE indiquées en rouge sont exposées à la surface des protéines virales lorsque les protéines adoptent leur conformation définitive à l'intérieur des protomères.

Les souches appartenant aux différents clades circulent de façon mondiale et l'isolement de souches apparentées dans des zones géographiques éloignées la même année indique une dynamique de transmission intercontinentale (figure 4). Le clade A comprend des souches isolées sur quatre continents entre 2004 et 2015. La grande majorité de souches responsables des épidémies de 2014 et 2016 sont apparentées au clade B. La lignée B1, majoritaire aux ÉtatsUnis en 2014, comprend également des souches isolées en Asie entre 2008 et 2014, et en Europe dont la France entre 2012 et 2014. La lignée B2, prédominante en Europe en 2014, est également représentée par des souches européennes circulant depuis 2009 (figure 4) et de rares souches américaines. Enfin, la lignée B3 fut majoritaire au cours des épidémies de 2016, à la fois aux États-Unis et en Europe, mais comprend aussi des souches isolées en Asie deux ans auparavant. Le clade $\mathrm{C}$, représenté par des souches isolées en Europe, aux États-Unis et en Asie entre 2004 et 2013, ne semble plus circuler actuellement. Enfin, le clade D comprend principalement des souches asiatiques isolées entre 2008 et 2010 et des souches plus récentes isolées également en Asie entre 2011 et 2013 ainsi qu'en Europe entre 2014 et 2016 (figure 4). Les souches d'EV-D68 de plusieurs lignées co-circulent au cours d'une même année et dans différentes régions géographiques, une caractéristique épidémiologique partagée avec d'autres types d'EV [10].
De même, le remplacement des lignées majoritaires lors de chaque épidémie a été décrit pour des EV d'autres espèces taxonomiques [10, 49].

\section{Comment les infections à EV-D68 peuvent-elles être surveillées?}

Le fait qu'à deux ans d'intervalle soient survenues deux épidémies de grande ampleur d'infections respiratoires souvent sévères avec atteintes neurologiques, justifie pleinement la nécessité de surveiller la circulation de l'EV-D68. Aux États-Unis et en Europe, la surveillance des infections à EV repose sur la notification volontaire des infections diagnostiquées chez les patients hospitalisés. La France bénéficie d'un réseau de surveillance des EV (RSE) mis en place depuis 1996 sous l'égide de Santé Publique France animé par les deux laboratoires du Centre National de Référence des Enterovirus et Parechovirus (Lyon et Clermont-Ferrand). Ce réseau, auquel participent 36 laboratoires de virologie hospitaliers, rapporte les données cliniques et virologiques via un site dédié (http://cnr.chu-clermontferrand.fr/CNR/). Cette surveillance porte en priorité sur les atteintes neuro-méningées 
et les infections néonatales, ce qui peut constituer une limite pour la surveillance spécifique des infections à EVD68 principalement associées à des infections respiratoires. L'existence du RSE permet toutefois de mettre en place une surveillance et des études collaboratives ciblées sur un sérotype en particulier. Ceci a ainsi permis de détecter des cas d'infections à EV-D68 en 2014 [8]. Le maintien de cette surveillance spécifique, dans les 2 laboratoires du CNR en France, a également permis la détection précoce des infections à EV-D68 en 2016.

La surveillance des infections à EV-D68 peut également s'appuyer sur celle des infections respiratoires réalisée par le réseau RENAL coordonné par le CNR Virus des infections respiratoires (dont la grippe) qui collecte de façon hebdomadaire le nombre de prélèvements testés pour la recherche des Picornavirus (incluant les EV et les rhinovirus) et le nombre de prélèvements positifs pour ces virus (https://www.grippenet.fr/fr/la-grippe/lasurveillance-en-france/). Le génotypage est cependant nécessaire pour détecter l'EV-D68 ou même les autres EV responsables d'infections respiratoires comme certains nouveaux types d'EV de l'espèce C. Par ailleurs, ce réseau a surtout pour vocation de suivre les épidémies respiratoires virales hivernales, en particulier associées aux virus grippaux, et les données hebdomadaires ne sont collectées que d'octobre à mai, ne couvrant pas complètement la période épidémique habituellement observée pour les infections à EV-D68 [8, 23, 28]. Le génotypage dans les infections respiratoires sévères ou en cas de recrudescence inhabituelle du nombre d'échantillons respiratoires positifs en été et au début de l'automne permettrait une détection précoce de la circulation de l'EV-D68 et une alerte rapide des cliniciens et des instances de Santé Publique en cas d'atteintes neurologiques sévères.

La surveillance syndromique peut également être un bon moyen de détecter les épidémies à EV-D68 et peut porter sur les infections respiratoires ou les MFA. Ainsi, par comparaison à la situation des années précédentes pour la même période, l'épidémie de 2014 dans l'État du Colorado (USA) s'est accompagnée d'une augmentation de $36 \%$ des passages aux urgences pour symptômes respiratoires et de $77 \%$ des hospitalisations associées à des signes respiratoires [37]. De façon inexpliquée, la circulation accrue de l'EV-D68, la même année en France, n'a pas été marquée par une recrudescence des hospitalisations ni une augmentation du nombre d'échantillons analysés positifs à EV-rhinovirus. Seule la mise en place d'une surveillance virologique ciblée, comprenant une analyse rétrospective d'échantillons respiratoires, a permis de détecter l'épidémie de 2014 [8]. La surveillance et la déclaration des cas de MFA peuvent également être réalisées mais ne permettent généralement une mise en évidence d'une circulation de
l'EV-D68 qu'a posteriori, les infections sévères n'étant souvent rapportées qu'au cours des épidémies. Cela permet toutefois une investigation plus poussée de ces atteintes neurologiques pour surveiller l'absence de circulation des poliovirus et identifier le type d'EV associé qui n'est pas toujours celui attendu. Début juin 2016, le CDC européen lançait une alerte concernant la recrudescence d'atteintes neurologiques sévères associées à l'EV-A71 en Espagne. En juillet, suite à l'alerte lancée par l'hôpital Necker concernant une recrudescence d'atteintes neurologiques sévères (dont des MFA) chez les enfants, Santé Publique France a mis en place un formulaire de déclaration des cas et les laboratoires du réseau ont été encouragés et aidés pour rapporter et investiguer de façon complète ces infections sévères. Entre mai et octobre 2016, 52 infections neurologiques sévères (encéphalites, myélites, cérébellite), associées à la détection du génome des $\mathrm{EV}$ dans au moins un prélèvement, ont été analysées chez des enfants (âge médian, trois ans). Si l'EV-A71 était, comme attendu, l'EV le plus fréquemment identifié, cette surveillance a permis de détecter neuf cas associés à l'EV-D68 dont une co-infection EV-A71/EVD68 [11]. Les cas de MFA doivent donc être reconnus par les cliniciens et la recherche des EV réalisée. Un effort pédagogique doit aussi être fait pour que la recherche des EV soit réalisée aussi dans les échantillons périphériques et pas seulement dans le LCR où elle est souvent négative.

\section{État des lieux et perspectives thérapeutiques}

Il n'existe pas de traitement standardisé des infections respiratoires sévères ou des complications neurologiques associées à l'EV-D68. Suite à l'identification du premier foyer de MFA, le CDC américain a réuni une équipe pluridisciplinaire d'experts pour établir des recommandations sur leur prise en charge clinique et thérapeutique. Il n'a pas été trouvé de preuves suffisantes d'efficacité des corticoïdes, des immunoglobulines ou des échanges plasmatiques bien que ces traitements immunomodulateurs ont souvent été administrés au cours de la prise en charge aiguë de cette pathologie [36]. Des essais cliniques randomisés sont nécessaires pour établir leur efficacité. Les trois antiviraux en développement (pleconaril, vapendavir et pocapavir) pour traiter les infections à EV-Rhinovirus n'ont pas d'activité in vitro sur les souches circulantes d'EV-D68. Une autre molécule, DAS181, développée sous la forme d'aérosol pour les infections à virus influenzae et parainfluenzae, lyse les acides sialiques liés en $\alpha 2,6$ et inhibe la réplication de l'EV-D68 in vitro à très faible dose. La fluoxétine est la seule molécule commercialisée (comme 
anti-dépresseur) ayant une activité in vitro en inhibant la réplication de l'ARN viral par sa liaison à la protéine $2 \mathrm{C}$ [50].

En l'absence de vaccin, la prévention repose sur le respect strict des règles d'hygiène universelle, et en particulier le lavage des mains, pour stopper la chaîne de transmission au sein de la famille et de la collectivité. Lorsqu'un patient est hospitalisé, l'équipe opérationnelle d'hygiène de l'établissement permettra la mise en place des mesures d'isolement de précaution dites 'contact' pour lutter contre la transmission manu portée et une prévention de type 'gouttelettes' pour prévenir la transmission respiratoire.

\section{Conclusion}

L'EV-D68 doit être considéré comme un pathogène émergent. Les épidémies de grande ampleur de 2014 et 2016, associées à des infections respiratoires parfois compliquées d'atteintes neurologiques sévères, doivent inciter à une surveillance continue. Si le génotypage exhaustif des échantillons respiratoires n'est pas possible en pratique courante, il faut l'encourager pour les infections sévères afin de détecter précocement la circulation de l'EVD68. L'investigation poussée des atteintes neurologiques sévères est également primordiale pour distinguer les EV des autres agents pathogènes possibles et doit porter sur la collection de multiples échantillons biologiques, le LCR n'étant pas contributif dans le cas des encéphalites ou paralysies associées aux EV. L'émergence de l'EV-D68 depuis 2008 illustre le potentiel épidémique et pathogène qui caractérise les EV. Depuis l'épidémie de 2014 et la crainte d'épidémies récurrentes, de nombreux efforts sont développés pour mieux connaître la physiopathologie de l'infection par ce virus et développer des thérapeutiques.

Remerciements. Nous remercions l'ensemble des participants au Réseau de surveillance des EV : Amiens (D Hecquet, C Segard), Angers (CT Tran, A Ducancelle, F Lunel-Fabiani), Bayonne (D Leyssene, A-C Jaouen), Besançon (Q Lepiller), Bordeaux (A Vilain-Parcé, ME Lafon, H Fleury), Brest (L Pilorgé, C Payan), Cahors (I Mendes Martin, N Wilhem), Caen (C Schanen, A Vabret), Dijon (K Balay, C Manoha, JB Bour), Frejus (T Hubiche, $\mathrm{P}$ del Giudice), Grenoble (C Morel-Baccard, J Lupo, P Morand), Haguenau (J Exinger), Lille (M Lazrek, D Hober), Limoges (S Rogez, S Alain), Lyon (I Schuffenecker, L Josset, G Billaud, B Lina), Marseille (L Ninove, R Charrel, X de Lamballerie), Montpellier (V Foulongne, M Segondy), Mulhouse (JM Delarbre), Nancy (S Berger, E Schvoerer), Nantes (M Coste-Burel), Nice (A Caramella, I Cannavo, V Giordanengo), Orléans (A Guigon, J Guinard),
Paris-Cochin (AS L'honneur, F Rozenberg), Paris-Necker (M Burgard, M Leruez), Paris-St Louis (M Salmona, J Legoff,), Paris-Trousseau (K Saloum, A Schnuriger), Poitiers (A Bourgoin, N Lévêque), Reims (L Andreoletti), Rennes (G Lagathu), Roanne (C Brechet), Rouen (V Lémée, M Gueudin, JC Plantier), St Etienne (S Pillet, B Pozzetto), Strasbourg (W Kack-Kack, S Fafi-Kremer), Toulouse (JM Mansuy, C Mengelle, J Izopet), Toulon-CHI (C Poggi), Toulon-HIA (F Janvier), Tours (K Stefic, C Gaudy, A Goudeau), Versailles (S Marque-Juillet), Villefranche (O Marchand, E Dorangeon).

Liens d'intérêts : Audrey Mirand déclare une « conférence sur invitation par la Société française de microbiologie et par la comité de la Ricai (réunion interdisciplinaire de chimiothérapie anti-infectieuse) ». Les autres auteurs déclarent ne pas avoir de lien d'intérêt en rapport avec cet article.

\section{Références}

1. Holm-Hansen CC, Midgley SE, Fischer TK. Global emergence of enterovirus D68 : a systematic review. Lancet Infect Dis 2016; 16 : e64-75.

2. Midgley CM, Jackson MA, Selvarangan R, et al. Severe respiratory illness associated with enterovirus D68 - Missouri and Illinois, 2014. Morb Mortal Wkly Rep 2014 ; 63 : 798-9.

3. Pastula DM, Aliabadi N, Hyanes AK, et al. Acute neurologic illness of unknown etiology in children-Colorado. August-September 2014. Morb Mortal Wkly Rep 2014;63:901-2.

4. Roux A, Lulu S, Waubant E, Glaser CA, Van Haren K. A polio-like syndrome in California : clinical radiologic and serologic evaluation of five children identified by a statewide laboratory over a twelve-month period. Neurology $2014 ; 82$ : P3.335.

5. Council of State Territorial Epidemiologists. CSTE position statement. In : Standardized case definition for acute flaccid myelitis. Atlanta GA : Council of State and Territorial Epidemiologists, 2015 (Consulté le 24/07/2017) http://c.ymcdn.com/sites/www.cste.org/resource/resmgr/ 2015PS/2015PSFinal/15-ID-01.pdf.

6. Marx A, Glass JD, Sutter RW. Differential diagnosis of acute flaccid paralysis ant its role in poliomyelitis surveillance. Epidemiol Rev $2000 ; 22: 296-316$.

7. Poelman R, Schuffenecker I, Van Leer-Butter C, et al. European surveillance for enterovirus D68 during the emerging North-American outbreak in 2014. J Clin Virol $2015 ; 71: 1-9$.

8. Schuffenecker I, Mirand A, Josset L, et al. Epidemiological and clinical characteristics of patients infected with enterovirus D68, France, July to December 2014. Euro Surveill 2016;21:30226.

9. Lang M, Mirand A, Savy N, et al. Acute flaccid paralysis following enterovirus D68 associated pneumonia, France, 2014. Euro Surveill $2014 ; 19: 20952$.

10. Solomon T, Lewthwaite P, Perera D, et al. Virology, epidemiology pathogenesis and control of enterovirus 71 . Lancet Infect Dis $2010 ; 10: 778-90$.

11. Antona D, Kossorotoff M, Schuffenecker I, et al. Severe paediatric conditions linked with EV-A71 and EV-D68, France, May to October 2016. Euro Surveill 2016;21:30402.

12. Knoester M, Schölvinck EH, Poelman R, et al. Upsurge of enterovirus D68, the Netherlands, 2016. Emerg Infect Dis 2017;23:140-3. 
13. Dyrdak R, Grabbe M, Hammas B, et al. Outbreak of enterovirus D68 of the new B3 lineage in Stockholm, Sweden, August to September 2016. Euro Surveill 2016;21:30403.

14. Barnabas C, Midgley S, Skov MN, et al. An enhanced enterovirus surveillance system allows identification and characterization of rare and emerging respiratory enteroviruses in Denmark, 2015-16. J Clin Virol 2017; $93: 40-4$.

15. Cabrerizo M, Garcia-Iniguez JP, Munell F, et al. First cases of severe flaccid paralysis associated with enterovirus D68 infection in Spain, 20152016. Pediatr Infect Dis 2017 ; 36 : 1214-6.

16. Messacar K, Robinson CC, Pretty K, Yuan J, Dominguez SR. Surveillance of enterovirus D68 in Colorado children reveals continued circulation. J Clin Virol 2017; $92: 39-41$.

17. Schieble JH, Fox VL, Lennette EH. A probable new human picornavirus associated with respiratory diseases. Am $J$ Epidemiol $1967 ; 85: 297-310$.

18. Peigue-Lafeuille H, Mirand A, Bailly JL, Henquell C. Entérovirus et parechovirus. EMC Maladies infectieuses 2016; 13:1-19.

19. Oberste MS, Maher K, Schnurr D, et al. Enterovirus 68 is associated with respiratory illness and shares biological features with both the enteroviruses and the rhinoviruses. J Gen Virol 2004 ; 85 : 2577-84.

20. Liu Y, Sheng J, Fokine A, et al. Structure and inhibition of EV-D68, a virus that causes respiratory illness in children. Science 2015 ; 347 : 71-4. 21. Baggen J, Thibaut HJ, Staring J, et al. Enterovirus D68 receptor requirements unveiled by haploid genetics. PNAS 2016;113:1399-404.

22. Liu Y, Shang J, Baggen J, et al. Sialic acid-dependent cell entry of human enterovirus D68. Nat commun $2015 ; 6: 8865$.

23. Imamura $T$, Oshitani $H$. Global reemergence of enterovirus D68 as an important pathogen for acute respiratory infections. Rev Med Virol $2015 ; 25: 102-14$

24. Blomqvist S, Savolainen C, Raman L, Roivainen M, Hovi T. Human rhinovirus 87 and enterovirus D68 represent a unique serotype with Rhinovirus and Enterovirus features. J Clin Microbiol 2002;40: 4218-23.

25. Hixon AM, Yu G, Leser JS, et al. A mouse model of paralytic myelitis caused by enterovirus D68. PLoS Pathog 2017; 13 : e1006199.

26. Smura T, Ylipaasto $P$, Klemola Pn Kaijalainen $S$, et al. Cellular tropism of human enterovirus D species serotypes EV-94, EV-70 and EV-D68 in vitro : implications for pathogenesis. J Med Virol 2010; 82 : 1940-9.

27. Wei $\mathrm{W}$, Guo H, Chang J, et al. ICAM-5/Telencephalin is a functional entry receptor for enterovirus D68. Cell Host Microbes 2016; 20: 631-41. 28. Midgley S, Watson JT, Nix WA, et al. Severe respiratory illness associated with a nationwide outbreak of enterovirus D68 in the USA (2014) : a descriptive epidemiological investigation. Lancet Respir Med $2015 ; 3: 879-87$.

29. Mertz D, Alawfi A, Pernica JM, et al. Clinical severity of pediatric respiratory illness with enterovirus D68 compared with rhinovirus or other enterovirus genotypes. CMAJ 2015; $187: 1279-84$

30. Moyer K, Wang H, Salamon D, Leber A, Meijas A. Enterovirus D68 in hospitalized children : sequence variation, viral loads and clinical outcomes. Plos One 2016; 11 : e0167111.

31. Meijer A, Van der Sanden S, Snijders BEP, et al. Emergence and epidemic occurrence of enterovirus 68 respiratory infections in the Netherlands in 2010. Virology $2012 ; 423: 49-57$.
32. Khetsuriani N, Lamonte-Fowlkes A, Oberste MS, Pallansch MA. CDC, Enterovirus surveillance-United States, 1970-2005. Morb Mortal Wkly Rep 2006; $55: 1-20$.

33. Kreuter JD, Barnes A, Mc Carthy JE, et al. A fatal central nervous system enterovirus 68 infection. Arch Pathol Lab Med 2011; 135 : 793-6. 34. Engelmann I, Fatoux M, Lazrek M, et al. Enterovirus D68 detection in respiratory specimens : association with severe disease. J Med Virol 2017;89: 1201-7.

35. PfeifferHC, Bragstas K, Skram MK, et al. Two cases of acute severe flaccid myelitis associated with enterovirus D68 infection in children, Norway, autumn 2014. Euro Surveill 2015;20:21062.

36. Sejvar JJ, Lopez AS, Corteses MM, et al. Acute flaccid myelitis in the United States-August-December 2014 : results of nation-wide surveillance. Clin Infect Dis 2016;63:737-47.

37. Messacar K, Schreiner TL, Maloney JA, et al. A cluster of acute flaccid paralysis and cranial nerve dysfunctino temporally associated with an outbreak of enterovirus D68 in children in Colorado, USA. Lancet $2015 ; 385: 1662-771$.

38. Maloney JA, Mirsky DM, Messacar K, et al. MRI findings in children with a cute flaccid paralysis and cranial nerve dysfunction occuring during the 2014 enterovirus D68 outbreak. AJNR Am J Neuroradiol $2015 ; 36: 245-50$.

39. Shen WC, Chiu HH, Chow KC, Tsai CH. MR imaging findings of enteroviral encephalomyelitis : an outbreak in Taiwan. AJNR Am J Neuroradiol $1999 ; 20: 1889-95$.

40. Martin JA, Messacar K, Yang ML, et al. Outcomes of Colorado childre with acute flaccid myelitis at 1 year. Neurology 2017;89:129-37.

41. Henquell C, Lina B. «Entérovirus ». In : Société française de microbiologie. Remic. 2015. pp. 629-34.

42. Bisseux M, Colombet J, Mirand A, et al. Monitoring human enteric viruses in wastewater and relevance to infections encountered in the clinical setting : results of a one-year experiment in Central France. Euro Surveill 2017 ; sous presse.

43. Ikeda T, Mizuta K, Abiko C, et al. Acute respiratory infections due to enterovirus 68 in Yamagata, Japan between 2005 and 2010. Microbiol Immunol 2012; $56: 139-43$.

44. Xiang Z, Li L, Ren L, et al. Seroepidemiology of enterovirus E68 infection in China. Emerg Microbes Infect 2017; 10: e32.

45. Tokarz R, Firth C, Madhi SA, et al. Worldwide emergence of multiple clades of enterovirus 68. J Gen Virol 2012;93:1952-8.

46. Du J, Zheng B, Zheng W, et al. Analysis of enterovirus 68 strains from the 2014 North American outbreak reveals a new clade, indicating viral evolution. Plos One 2015; 10 : $\mathrm{e} 0144208$

47. Xiang Z, Xie Z, Liu L, et al. Genetic divergence of enterovirus D68 in China and the United States. Sci Rep 2016; 6 : 27800.

48. Tan Y, Hassan F, Schuster JE, et al. Molecular evolution and intraclade recombination of enterovirus D68 during the 2014 outbreak in the United States. J Virol 2016; 90 : 1997-2007.

49. Hassel C, Mirand A, Lukashec A, et al. Transmission pattern of human enterovirus 71 to, from and among European countrier, 2003 to 2013. Euro Surveill $2017 ; 20: 30005$.

50. Rhoden E, Zhang M, Nix WA, Oberste MS. In Vitro efficcacy of antiviral compounds against enterovirus D68. Antimicrob Agents Chemother $2015 ; 59: 7779-81$. 